\title{
Moving Forward from the "Boot Camp Method" To Learning-Curve Development in Simulation-Based Paracentesis Training For Undergraduate Medical Students
}

\author{
Martin Inzunza \\ Pontificia Universidad Católica de Chile \\ Rodrigo Tejos \\ Pontificia Universidad Católica de Chile \\ Ignacio Villagrán \\ Pontificia Universidad Católica de Chile \\ Eduardo Fuentes \\ Pontificia Universidad Católica de Chile \\ Caterina Contreras \\ Pontificia Universidad Católica de Chile \\ Javier Vela \\ Pontificia Universidad Católica de Chile \\ Margarita Pizarro \\ Pontificia Universidad Católica de Chile \\ Adnan Alseidi \\ University of California, San Francisco \\ Julian Varas \\ Pontificia Universidad Católica de Chile
}

Arnoldo Riquelme ( $\nabla$ a.riquelme.perez@gmail.com )

Pontificia Universidad Católica de Chile

\section{Research Article}

Keywords: Abdominal paracentesis, Simulation-based mastery learning (SBML), boot-camp method

Posted Date: March 11th, 2021

DOl: https://doi.org/10.21203/rs.3.rs-276089/v1

License: (9) This work is licensed under a Creative Commons Attribution 4.0 International License. Read Full License 
Page 2/16 


\section{Abstract}

Background: Abdominal paracentesis is an essential competence for physicians. Simulation-based mastery learning (SBML) programs lead to developing these skills for medical students. Most programs are structured as short boot-camps, without assessing skills' retention mid and long-term or learning curves.

Aim: To assess the learning curve of paracentesis through an SBML program for medical students and compare this learning curve-based program with the boot-camp method.

Methods: A prospective quasi-experimental study was conducted. A cohort of medical students participated in an SBML program with successive sessions until proficiency criteria were met while their learning curve was assessed (LC group). A control group received an SBML boot-camp intervention (BC group). As a proficient group, gastroenterology fellows (GF group) performed a paracentesis on the simulated model. The skills of the three groups were compared using technical/proficiency scores. Learning curves and cost analysis were performed.

Results: $100 \%$ of the LC group achieved proficiency in the fourth session, with a flattening learning curve between third-fourth sessions. Comparing the initial and final sessions of LC group showed a significant improvement in their scores. When comparing the three groups, statistical differences were found in the skill scores, with the BC group having the lowest. The overall cost per participant was highest for the LC group.

Conclusion: This study identifies a learning curve for paracentesis with an SBML program. The LC group significantly improved their skills, comparable with the GF group's performance. SBML focused on a learning curve ensured better skills acquisition than the "boot-camp method."

\section{Introduction}

Ascites is a major complication of liver cirrhosis, occurring in up to $50 \%$ of patients [1]. Its development reflects the progression of the disease and is associated with substantially increased mortality [2]. More than 100,000 inpatient admissions for complicated liver cirrhosis occur in the U.S. annually [1]. Bedside paracentesis (diagnostic and/or therapeutic) is one of the cornerstones in the management of patients with ascites. Paracentesis performed early in hospital admission is associated with increased short-term survival [3].

Thus, paracentesis is a significant competence for physicians worldwide. As with other medical procedures, skill is gained through frequent performance of the procedure on patients, according to Halsted's traditional medical learning method [4]. However, exposure to the procedure could be infrequent or non-standardized during the clinical education process, since exposure to patients is not always homogeneous. In consequence, paracentesis is often performed by learners who are not proficient or confident $[5,6]$, which creates a risk for adverse events. According to the literature, a complication rate of 
$1.6 \%$ has been reported, including bowel perforation, hemorrhage, hemoperitoneum, and puncture site infection $[4,7,8]$.

Simulation-based mastery learning (SBML) with deliberate practice provides the opportunity for medical students and residents to develop procedural skills $[6,9]$. In recent years, different simulation-based programs have been designed and assessed for paracentesis training $[1,4,6,10,11]$, supporting their effectiveness in skills acquisition. In addition, accreditation entities now demand simulation training for students, residents, and fellows [12].

Most of these programs are structured as short boot camps, meaning a brief educational session is immediately followed by an evaluation without assessing skills' retention mid and long-term $[6,10,13$, 14]. Although these programs have led to changes in medical education curricula worldwide, the failure to assess mid and long-term acquisition of the desired skills induces a bias concerning the number of training sessions required to master a procedure. However, understanding the learning curve of this procedure could clarify the actual number of training sessions required to achieve proficiency.

This study aims to assess the learning curve of abdominal paracentesis through an SBML program for medical students and to compare two different learning methodologies for paracentesis skills acquisition: the boot camp method and the learning curve-based method.

\section{Methods}

\section{Study design and participants}

A prospective quasi-experimental study was designed and conducted.

Medical students from the Pontificia Universidad Católica de Chile were recruited between 2018 and 2019 to participate in this study, according to our predefined inclusion and exclusion criteria (Table 1).

The first cohort of students were recruited from March to December 2018. This group was defined as the Learning Curve group (LC). The control group was recruited from March to December 2019. This group was defined as the boot camp group (BC).

As a proficient group, gastroenterology fellows (GF) were recruited. They had not received mandatory simulation-based training in paracentesis during their medical education; rather, reflecting the traditional medical learning method without simulation [4], they had practiced directly on a minimum of 20 real patients to ensure adequate experience according to the Core Curriculum recommendation of the Gastroenterology Leadership Council [12]. They were recruited according to our inclusion and exclusion criteria (Table 1).

All participants agreed to participate voluntarily, and informed consent was obtained.

This study was approved by the Ethics Committee of the Pontificia Universidad Católica de Chile in compliance with the Declaration of Helsinki. 


\section{Simulated paracentesis model}

A previously validated simulated paracentesis model was used in this project $[4,15]$. The model was designed and developed by a group of experts (gastroenterologists and surgeons) together with specialist designers of simulated models at Pontificia Universidad Católica de Chile. The validation process included a qualitative analysis of how well the model met clinical educational needs, including fidelity, washing capability, transportation, reuse capacity, low cost, safety for students, and use of the Z-traction technique on the abdominal skin (Fig. 1). The innovative characteristics of this paracentesis model led to a patent application, with an overall cost of $\$ 1,000$ USD for each model [15].

\section{Simulation-based training program}

Both the $\mathrm{LC}$ and $\mathrm{BC}$ groups received previously validated educational support material developed by clinical experts [4]. This material included a video tutorial on the paracentesis technique, encompassing theoretical and practical concepts. The participants were asked to review the educational support material prior to the training sessions. To ensure a homogeneous framework, this educational material, including the video tutorial, was reviewed in a 15-minute briefing by the clinical instructor at the beginning of both the $\mathrm{LC}$ and the $\mathrm{BC}$ training program.

The LC group underwent successive training sessions using the simulated paracentesis model and were tutored by an expert who provided them with direct feedback.

The participants attended a half-hour training session once a week until they met the pre-established criteria for proficiency.

The purpose of this format was to reduce any possible forgetfulness bias and, at the same time, to avoid conducting all the training sessions on one day.

The BC group underwent a one-day SBML boot camp program that consisted of four hours of paracentesis training with the simulated model. The workshop was performed in groups of four participants and lead by an expert tutor who provided individual feedback. The students were encouraged to observe and evaluate the performance of their peers [4].

The GF group received the same educational material to standardize the theoretical-practical framework. Following the 15-minute briefing with the video tutorial, they performed a single paracentesis puncture on the simulated model.

Debriefing at the end of the training program was provided by an expert tutor who provided feedback to the three groups (Fig.

2).

\section{Assessment tools}


All the sessions for each group were video recorded. The video-recordings were randomly analyzed by a blinded expert who did not participate in any other training or educational activity. The expert evaluated the video-recordings based on technical skills and specific procedural milestones. The primary outcomes were the OSATS (objective structured evaluation of technical skills) score [16] and the DOPS (direct observation of procedure skills) score. A previously modified and internationally validated OSATS scale was used $[17,18]$ (Table 2). The DOPS scale was adapted by an experts' panel $(n=8)$ to assess compliance with the specific requirements of the paracentesis procedure (Table 3 ). Both assessment tools were available to participants to guide their learning.

At the end of both training programs, a seven-item survey was administered to participants to assess their perception of their performance. Each question comprised a five-point Likert scale. This instrument was previously designed and validated by Barsuk and adapted to Spanish by Tejos et al. [4, 6] (Table 4).

\section{Proficiency criteria}

We established a minimum passing score for paracentesis clinical skills determined with the input of eight clinical experts using the modified Angoff standard-setting point [12, 19]. For the modified OSATS scale, the minimum passing score was set at 23 out of 25 points (equivalent to $92 \%$ of the maximum score), and for the DOPS scale, the minimum score was set at 25 out of 27 points (equivalent to $93 \%$ of the maximum score). The maximum time to perform the procedure was set at 20 minutes.

The LC group were informed at the beginning of the training program that the main objective was the acquisition of skills. If a student did not achieve the minimum required score within a month, they had to continue training until they achieved proficiency. For the BC group, if a participant did not achieve the required score, they were required to attend a second workshop with a different group of peers.

\section{Learning curve analysis}

Mixed-effects models with a random intercept were constructed to analyze differences in the consecutive OSATS and DOPS scores of each trainee. Since scores from consecutive training sessions for the same subject were compared, an intra-subject correlation was expected, which would produce biased estimates of the standard errors when estimated using linear regressions models [20]. Mixed-effects models can be used to estimate standard errors that take the clustering within subjects into account and have been proven to have higher statistical power than conventional repeated-measures analysis of variance [21]. Also, pairwise comparisons were made between adjacent sessions results, with multiple comparisons being adjusted by Bonferroni's correction (to control type I error).

The mixed-effects models allowed an analysis of the LC group [22] that represented the trainees' average OSATS and DOPS scores. Each trainee's trajectory, showing the trainee's individual learning curve, was estimated using Growth Curve Modeling [23], specifying an intercept and a random coefficient model.

Given that the mixed-effects models' residuals did not have a normal distribution, the standard error was estimated using bootstrapping (10,000 replications). Thus, $95 \%$ confidence intervals (Cl) were obtained 
using the bias-corrected and accelerated method [24]. Mean scores and 95\% Cl were expressed for each training session.

\section{Cost-analysis}

A cost-analysis comparison between the two training program methodologies was carried out. Medical supplies, infrastructure, simulated model and teaching-time costs were analyzed (Table 5).

\section{Statistical analysis}

All analyses were performed using STATA version 16 (StataCorp LLC, College Station, TX, USA). Learning curve statistical analysis was detailed previously. Mann-Whitney U and Kruskal-Wallis tests were performed to compare the groups. All results were expressed in terms of median and IQR or mean \pm SD, as appropriate. A p-value of $<0.05$ was considered statistically significant.

\section{Results}

\section{Participants}

Two-hundred-and-thirty-one medical students were invited to participate between 2018 and 2019, out of which 71 were included in the study (a 30.7\% acceptance rate). The global mean age was $23 \pm 1,4$ and $48 \%$ of the participants were female.

Twenty-one students were included in the LC group, 50 students were included in the BC group, and 10 gastroenterology fellows comprised the proficient group (the GF group). Two participants in the LC group did not complete all the training sessions and were excluded from the analysis (Table 6).

\section{Learning curve group (LC) performance}

The OSATS scores (Fig. 3a) showed significant differences $(p<0.001)$ between the first session (mean score 18.58 [95\% Cl 17.63-19.52]) and the second session (mean score 21.89 [95\% Cl 21.35-22.43]), between the second and third session (mean score 23.47 [95\% Cl 23.02-23.93], p< 0.001), and between the third and fourth session (mean score 24.37 [95\% Cl 23.90-24.84], p < 0.05). Individual trajectories can be observed in Fig. 3b.

The DOPS scores did not show significant differences $(p=0.151)$ between the second session (mean score 25.16 [95\% Cl 24.68-25.64]) and the third session (mean score 25.84 [95\% Cl 25.33-26.36]) or between the third and fourth session (mean score 26.47 [95\% Cl 26.07-26.88], p = 0.167) (Fig. 4a). However, there were significant differences between the first session (mean score 21.53 [95\% Cl 20.55$22.50]$ ) and the second session ( $p<0.001)$, between the first session and the third session $(p<0.001)$, and between the first session and the fourth session $(p<0.001)$. Individual trajectories can be observed in Fig. 4b. The learning curve inflection point took place in the second session. However, the majority of the trainees continued to improve in the third session. 
All of the participants in the LC group had met the proficiency criteria by the fourth session (LC4). Interestingly, $84 \%$ of the participants met the proficiency criteria in terms of OSATS and $89 \%$ in terms of DOPS in the third session of the training program.

\section{Boot camp group (BC) performance}

Only $66 \%$ of the BC group met the proficiency criteria as measured by OSATS, and only $86 \%$ achieved the DOPS minimum required score. Therefore, 17 students had to repeat the workshop. The median OSATS and DOPS scores were 25 points (24-25) and 27 points (26-27), respectively (Fig. 5).

\section{Gastroenterology fellows' (GF) performance}

Ninety percent of the fellows met the proficiency criteria, with a median score for OSATS and DOPS of 25 points (24-25) and 26 points (26-27), respectively. Surprisingly, one of the fellows did not achieve the required minimum OSATS and DOPS scores, achieving 23 and 24 points, respectively (Fig. 5).

\section{Impact of the learning curve-based training program (LC group)}

We found no statistically significant differences in the participants' OSATS and DOPS scores between the fourth training session (LC4) of the LC group and the GFs' performance (OSATS: 24 points [24-25] vs. 25 points (24-25), $p=0.802$ ] and DOPS: 27 points [26-27] vs. 26 points $(26-27), p=0.446)$, respectively.

However, the LC4 and GF OSATS results were significantly superior to those of the BC group (24 points [24-25] vs. 25 points [24-25] vs. 24 points [21-25], respectively; $p=0.042]$. No differences were found in the DOPS scores between the three groups (Fig. 5).

\section{Assessment of the training program perception}

The questionnaire administered to participants showed that they highly valued both training programs, based on a 5-point Likert scale, where $1=$ strongly disagree and $5=$ strongly agree. The LC group reached a mean of $4.9 \pm 0.26$ points, and the BC group achieved a mean of $4.8 \pm 0.39$ points, with no statistical differences $(p=0.621)$ (Table 4$)$.

\section{Cost analysis}

The total cost for the LC group was $\$ 121$ USD per student for all four sessions; thus, the cost of each session was $\$ 30$ USD per student. The total cost of the boot camp was $\$ 80$ USD per student. The cost of the teaching time was the most expensive item for each group (Table 5).

\section{Discussion}

We found that proficiency was achieved by $100 \%$ of the LC group at the fourth session (LC4). We found no differences when comparing the LC4 and the GF group's OSATS and DOPS scores. Both the LC and GF 
groups had significantly superior OSATS results compared to the BG group; there were no differences in the DOPS scores between the three groups.

The main strength of this work is that we evaluated the students' performance by analyzing their learning curve. This is a very important strength of this study, since one of the main confounding biases in previous educational studies has been the focus on evaluating the outcome of education after conducting just a "point in time" training, exemplified by the boot camp training. Another strength of our study is the high quality of the study design. Specifically, we believe that the experimental interventions, the blinded experts who reviewed the videos, and the validated scales used to measure the outcomes give strong internal validity to our work.

One of the main limitations of this study is the absence of randomization. This problem was considered during the design and planning of the study; however, we decided to go with a quasi-experimental design because we were not able to randomize the gastroenterology fellows nor the medical students as they were recruited one by one during different periods.

Another limitation is that the simulated model made in our institution does not contain echogenic materials; thus, the paracentesis was performed using classic anatomical repairs. However, this is compatible with local reality, where only a minority of punctures are ultrasound-guided. We also believe that the absence of paracentesis training on a real patient and the evaluation of the learning curve for this procedure could be an additional limitation; nevertheless, it seems unethical to train medical students on real patients due to the strong evidence supporting the use of simulation as a method of learning technical skills. Moreover, as we noted in our results, even clinical experience of 20 or more procedures without previous standardized training does not guarantee proficiency $[6,12]$.

Knowing and understanding the learning curves of medical and surgical procedures is essential to designing effective training programs and defining the minimum competencies for medical education $[17,25]$. Therefore, the purpose of this study was to determine the learning curve in acquiring the technical skills to perform paracentesis in a simulated model and compare it with the performance of students trained by the widely accepted boot camp method. We also compared both groups with gastroenterology fellows, who are used to executing this procedure during their daily clinical practice.

Our undergraduate medical students trained through the LC program achieved a level of procedural proficiency comparable to the performance of more experienced professionals, that is, the gastroenterology fellows, who have four to five years of postgraduate training. Conversely, the students trained through the boot camp method (BC) had statistically significant worse results in terms of the skills they acquired, and $34 \%$ did not reach the required proficiency level at the end of their training. Moreover, one of the gastroenterology fellows did not meet the proficiency criteria in his performance, reflecting the importance of standardized training.

The results of this study allow us to claim that a paracentesis simulation-based training program requires at least three to four practical sessions for students to achieve proficiency. Students acquire technical 
skills progressively and in a standardized methodological sequence, whereas in the boot camp method, they are asked to recall procedural steps seen in a video just a few minutes previously [6]. Thus, we recommend moving from the one-day boot camp method in SBML to short, successive training sessions with deliberate practice on a simulated model, which results in progressive learning $[10,13]$.

Although the overall cost per student of the LC intervention is higher, one-third of the students in the BC group had to repeat the workshop, which was not factored into the cost analysis. Regardless of the learning methodology, the highest cost was related to the teaching time (Table 5).

In future studies, alternatives to optimize teaching and feedback can be explored, such as tele-mentoring. Quezada et al. used this methodology to teach advanced laparoscopic surgical skills through a mobile app, with remote feedback provided by expert tutors [26]. This novel method could optimize the teaching time and has been proved as effective as in-person instruction [26]. Also, current studies on simulationbased programs and medical/surgical skills still focus primarily on levels 1 and 2 of the Kirkpatrick model for learning evaluation and educational impact $[4,15,27,28]$. More research is needed on levels 3 (transfer of skills to real patients) and 4 (costs and service quality).

In conclusion, this study outlines a learning curve for the paracentesis procedure in a simulated model and demonstrates that a SBML program based on that learning curve improves technical skills significantly in medical students. Also, the performance of the students trained with this method was comparable with that of gastroenterology fellows with much more clinical experience.

\section{Declarations}

\section{AUTHOR CONTRIBUTIONS STATEMENT}

M.I., R.T., M.P., J.Va., and A.R. designed the study. M.I., R.T., and I.V. recruited the participants. M.I., I.V., C.C., J.Ve., and M.P. conducted the experiments. M.I., E.F., A.A., J.Va., and A.R. analyzed the results and performed the statistical analysis. M.I. and R.T. drafted the manuscript; all authors reviewed the manuscript.

\section{ADDITIONAL INFORMATION}

Competing Interests Statement: The authors declare no competing interests.

This work is part of the Master of Health Science thesis authored by Martin Inzunza.

\section{References}

1. Corona, J. et al. Ascites module for third-year medical students: group-based learning workshop on the diagnosis, workup, and management of ascites. MedEdPORTAL J Teach Learn Resour. 13, 10546 https://doi.org/10.15766/mep_2374-8265.10546 (2017). 
2. D’Amico, G., Garcia-Tsao, G. \& Pagliaro, L. Natural history and prognostic indicators of survival in cirrhosis: a systematic review of 118 studies. J Hepatol. 44 (1), 217-231 (2006).

3. Orman, E. S., Hayashi, P. H., Bataller, R. \& Barritt, A. S. Paracentesis is associated with reduced mortality in patients hospitalized with cirrhosis and ascites. Clin Gastroenterol Hepatol. 12 (3), 496503 (2014).

4. Tejos, R. et al. Simulated training program in abdominal paracentesis for undergraduate medical students. Gastroenterol Hepatol. 42 (4), 239-247 (2019).

5. Huang, G. C. et al. Beyond the comfort zone: residents assess their comfort performing inpatient medical procedures. Am J Med. 119 (1), 7117-7124 (2006).

6. Barsuk, J. H. et al. Simulation-based education with mastery learning improves paracentesis skills. $J$ Grad Med Educ. 4 (1), 23-27 (2012).

7. De Gottardi, A. et al. Risk of complications after abdominal paracentesis in cirrhotic patients: a prospective study. Clin Gastroenterol Hepatol. 7 (8), 906-909 (2009).

8. Sharzehi, K., Jain, V., Naveed, A. \& Schreibman, I. Hemorrhagic complications of paracentesis: a systematic review of the literature. Gastroenterol Res Pract 2014, 985141; 10.1155/2014/985141 (2014).

9. Ericsson, K. A. Deliberate practice and the acquisition and maintenance of expert performance in medicine and related domains. Acad Med. 79 (10), S70-81 (2004).

10. Wayne, D. B. et al. Progress toward improving medical school graduates' skills via a "boot camp" curriculum. Simul Healthc. 9 (1), 33-39 (2014).

11. Sall, D. et al. Paracentesis simulation: A comprehensive approach to procedural education. MedEdPORTAL J Teach Learn Resour. 14, 10747 https://doi.org/10.15766/mep_2374-8265.10747 (2018).

12. Ryen, R. et al. Training the gastroenterologist of the future: the gastroenterology core curriculum. Gastroenterology. 124 (4), 1055-1104 (2003).

13. Wayne, D. B., Barsuk, J. H., O'Leary, K. J., Fudala, M. J. \& McGaghie, W. C. Mastery learning of thoracentesis skills by internal medicine residents using simulation technology and deliberate practice. J Hosp Med. 3 (1), 48-54 (2008).

14. Barsuk, J. H. et al. The effect of simulation-based mastery learning on thoracentesis referral patterns. J Hosp Med. 11 (11), 792-795 (2016).

15. Uslar, T. et al. Diseño e implementación de un modelo de enseñanza en ambiente simulado de paracentesis abdominal. ARS MEDICA Rev Ciencias Médicas. 42 (2), 34-41 (2017).

16. Martin, J. A. et al. Objective structured assessment of technical skill (osats) for surgical residents. $\mathrm{Br}$ J Surg. 84 (2), 273-278 (1997).

17. Varas, J. et al. Significant transfer of surgical skills obtained with an advanced laparoscopic training program to a laparoscopic jejunojejunostomy in a live porcine model: feasibility of learning advanced laparoscopy in a general surgery residency. Surg Endosc. 26 (12), 3486-3494 (2012). 
18. Aggarwal, R. et al. Skills acquisition for laparoscopic gastric bypass in the training laboratory: an innovative approach. Obes Surg. 17 (1), 19-27 (2007).

19. De Champlain, A. F. (2018). Standard setting methods in medical education in Understanding medical education (eds. Swanwick, T., Forrest, K. \& O'Brien, B.C.) 347-359 (Wiley-Blackwell, 2018).

20. Rabe-Hesketh, S. \& Skrondal, A. Multilevel and longitudinal modeling using Stata(Stata Press, 2012).

21. Ma, Y., Mazumdar, M. \& Memtsoudis, S. G. Beyond repeated-measures analysis of variance: advanced statistical methods for the analysis of longitudinal data in anesthesia research. Reg Anesth Pain Med. 37 (1), 99-105 (2012).

22. Howard, N. M., Cook, D. A., Hatala, R. \& Pusic, M. V. Learning curves in health professions education simulation research: A systematic review. [published online ahead of print, 2020 Jul 15]. Simul Healthc 2020;10.1097/SIH.0000000000000477.

23. Rabe-Hesketh, S. Growth-curve models in Multilevel and longitudinal modeling using Stata (eds. Rabe-Hesketh, S. \& Skrondal, A.) 343-376 (Stata Press, 2012).

24. Carpenter, J. \& Bithell, J. Bootstrap confidence intervals: when, which, what? a practical guide for medical statisticians. Stat Med. 19 (9), 1141-1164 (2000).

25. Hopper, A. N., Jamison, M. H. \& Lewis, W. G. Learning curves in surgical practice. Postgrad Med J. 83 (986), 777-779 (2007).

26. Quezada, J. et al. Minimally invasive tele-mentoring opportunity: the mito project. Surg Endosc. 34 (6), 2585-2592 (2020).

27. Kirkpatrick, D. L. \& Kirkpatrick, J. D. Evaluating training programs: The four levels. (Berrett-Koehler 1994).

28. Kirkpatrick, D. Techniques for Evaluating Training programs: Revisiting Kirkpatrick's four-level model. Train Dev. 50 (1), 54-59 (1996).

\section{Tables}

Due to technical limitations, the tables are only available as a download in the supplemental files section.

\section{Figures}




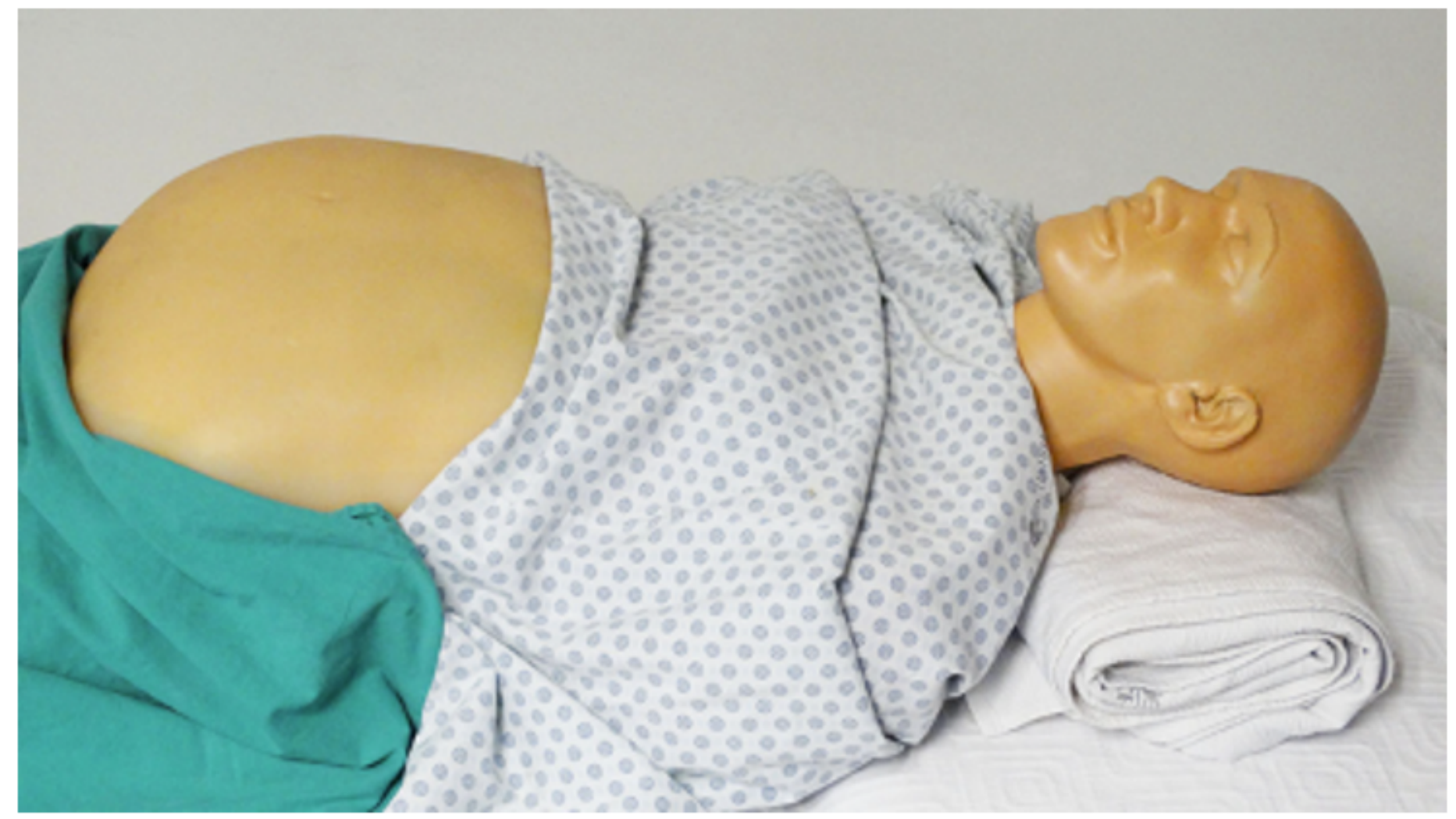

Figure 1

Abdominal paracentesis simulated model (from Tejos et al.) [4].

\begin{tabular}{|c|c|c|c|}
\hline & $\begin{array}{l}\text { LEARNING CURVE GROUP } \\
(\mathrm{n}=19)\end{array}$ & $\begin{array}{l}\text { BOOT CAMP GROUP } \\
(n=50)\end{array}$ & $\begin{array}{l}\text { GASTROENTEROLOGY } \\
\text { FELLOWS GROUP } \\
(\mathbf{n = 1 0 )}\end{array}$ \\
\hline $\begin{array}{l}\text { STAGE I: } \\
\text { BRIEFING }\end{array}$ & & & \\
\hline $\begin{array}{l}\text { STAGE II: } \\
\text { TRAINING WITH } \\
\text { DELIBERATE } \\
\text { PRACTICE }\end{array}$ & & & \\
\hline $\begin{array}{l}\text { STAGE III: } \\
\text { DEBRIEFING }\end{array}$ & 跑 & 筂 & 泟 \\
\hline
\end{tabular}


Figure 2

Workflow for each group in the simulation-based paracentesis training program
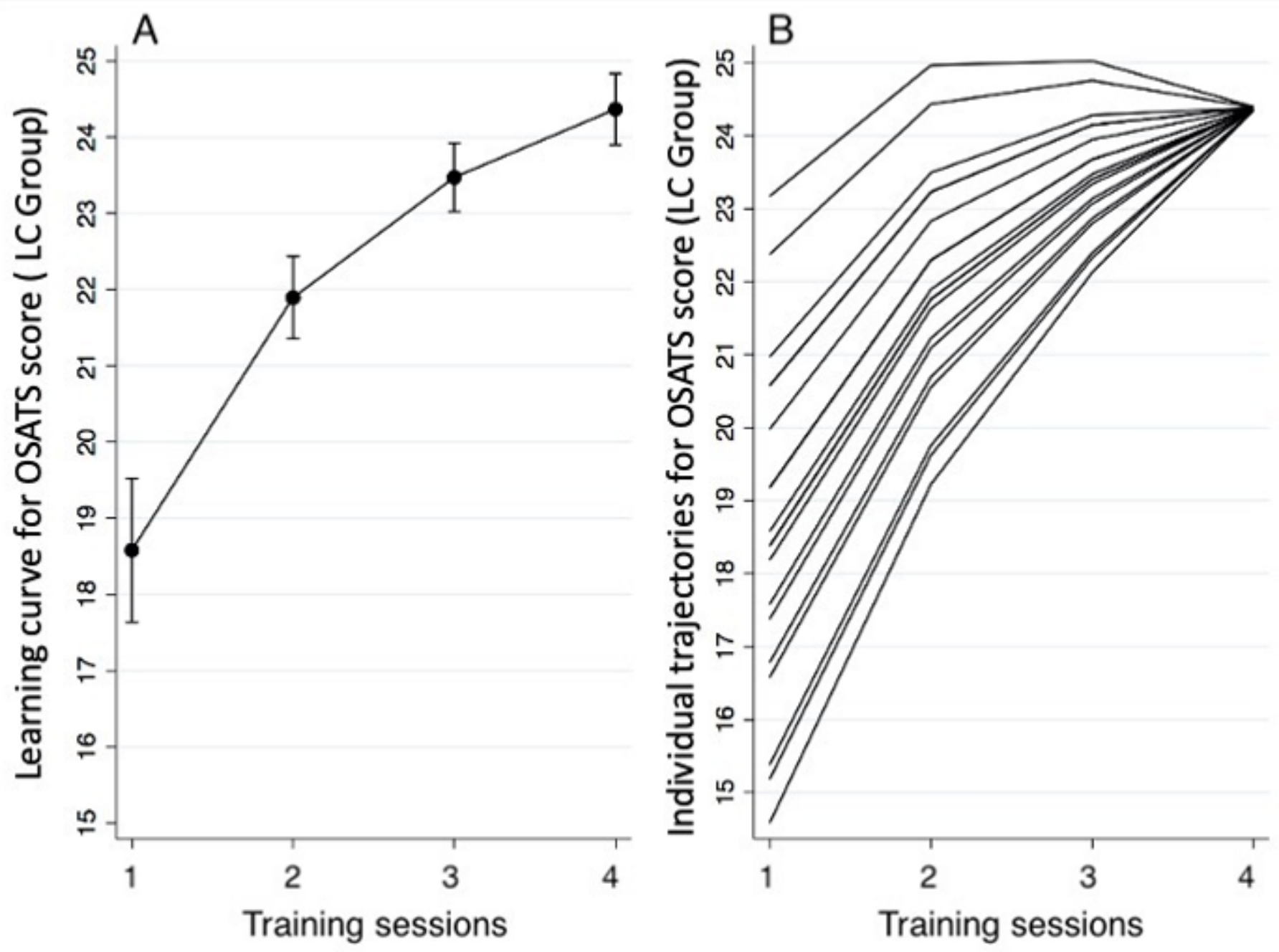

Figure 3

a: Learning curve based on the modified OSATS scores of the LC group. Dot plot with mean and 95\% confidence interval for every training session. b. Individual trajectories for each participant in the LC group. 

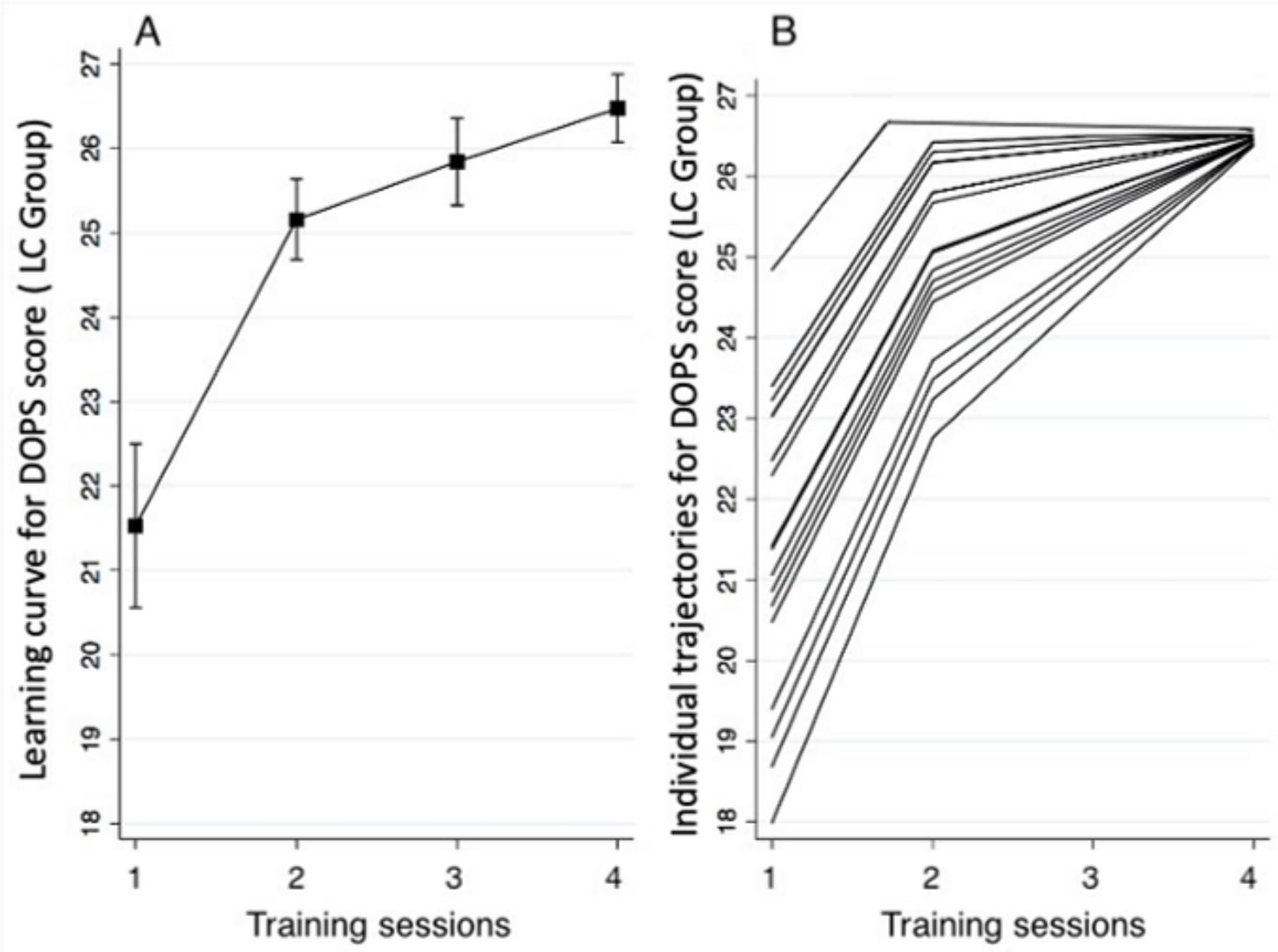

Figure 4

a: Learning curve based on the DOPS scores of the LC group. Dot plot with mean and $95 \%$ confidence interval for every training session. b: Individual trajectories for each participant in the LC group. 

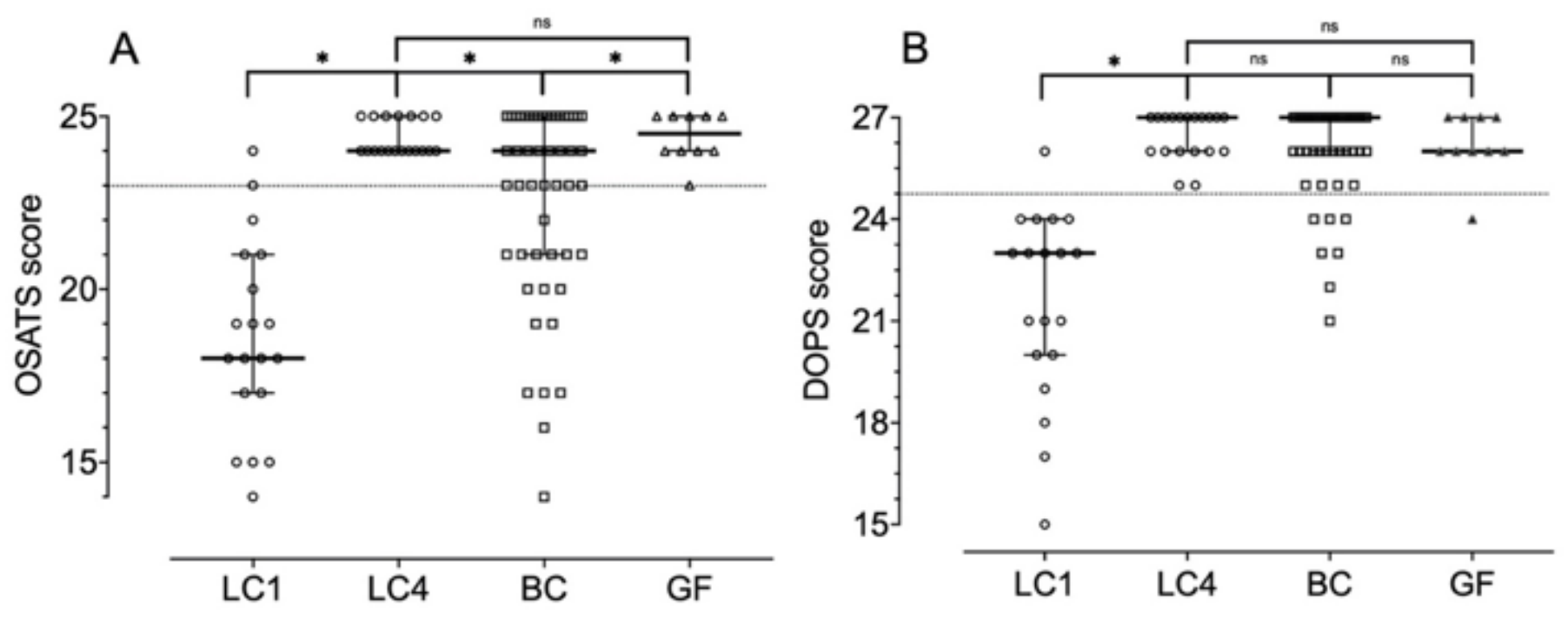

Figure 5

a: Comparison of modified OSATS scores between learning-curve group session 1 (LC1), session 4 (LC4), boot camp group (BC), and gastroenterology fellows group (GF). b: DOPS score comparison. Scattered plot: median and interquartile range or mean and SD, as appropriate. Dotted line $=$ cutoff score. $*=$ statistically significant. ns $=$ not statistically significant.

\section{Supplementary Files}

This is a list of supplementary files associated with this preprint. Click to download.

- Tables.docx 